Hossein M. Shodja* and Ehsan Rashidinejad

\title{
Interacting functionally graded quantum wires/ quantum dots with arbitrary shapes and general anisotropy within a distinct piezoelectric matrix
}

\begin{abstract}
An accurate determination of the two- and threedimensional electro-elastic fields of periodically as well as arbitrarily distributed interacting quantum wires (QWRs) and interacting quantum dots (QDs) of arbitrary shapes within a piezoelectric matrix is of particular interest. Both the QWR/QD and the barrier may be made of materials with distinct general rectilinear anisotropy in elastic, piezoelectric, and dielectric constants. The lattice mismatch between the $\mathrm{QWR} / \mathrm{QD}$ and the barrier is accounted by prescribing an initial misfit strain field within the QWR/QD. Previous analytical treatments have neglected the distinction between the electro-mechanical properties of the QWR/ $\mathrm{QD}$ and those of the barrier. This simplifying assumption is circumvented in the present work by using a novel electromechanical equivalent inclusion method in Fourier space (FEMEIM). Moreover, the theory can readily treat cases where the QWRs/QDs are multiphase or functionally graded (FG). It was proven that for two-dimensional problems of either a periodic or an arbitrary distribution of FG QWRs in a transversely isotropic piezoelectric barrier, the elastic and electric fields are electrically and elastically impotent, respectively, and no electric field would be induced in the medium provided that the rotational symmetry and polarization axes coincide. Some numerical examples of more frequent shapes and different distributions of indium nitride QDs/QWRs within transversely isotropic aluminum nitride barrier are solved.
\end{abstract}

Keywords: anisotropic piezoelectric media; electromechanical inclusion; Fourier space; quantum wires; quantum dots.

DOI 10.1515/jmbm-2014-0001

\footnotetext{
*Corresponding author: Hossein M. Shodja, Department of Civil Engineering, Sharif University of Technology, 11155-9313 Tehran, Iran, e-mail: shodja@sharif.edu; and Institute for Nanoscience and Nanotechnology, Sharif University of Technology, 11155-9161 Tehran, Iran
}

Ehsan Rashidinejad: Department of Civil Engineering, Sharif University of Technology, 11155-9313 Tehran, Iran

\section{Introduction}

Currently, the excellent nanotechnological applications of the group III multifunctional nitrides such as aluminum nitride (AlN) and indium nitride (InN) have drawn the attention of the scientific and industrial communities of diverse disciplines. These materials can be used to grow quantum structures (QSs) including quantum wires (QWRs) and quantum dots (QDs), which play a vital role in the developments of novel devices. Epitaxially grown and chemically synthesized semiconductor QDs have received much attention in recent years. Cadmium selenide (CdSe) and indium arsenide (InAs), which are classified as II-VI and III-V semiconductors, respectively, are utilized in the fabrication of core-shell semiconductor nanocrystal QDs by chemical synthesis. The core-shell semiconductors consisting of CdSe and zinc selenide ( $\mathrm{ZnSe}$ ) have immense optoelectronic properties in the blue-green spectral regions. The attractive optical and physical properties of QDs have made them very valuable, in particular, for biomedical and bioengineering applications; for example, for labeling and tracking proteins, genes, and cells of interest as well as cancer diagnosis and therapy among other applications. Core-shell QDs are used in solar cells as absorbing photovoltaic components to enhance the light absorbance and increase the efficiency of the solar cells. To further increase the efficiency of the photovoltaic cells, core-shell QDs with functionally graded (FG) shells are used; FG shells are used to help remove the interfacial defects and improve the processes of the charge transfer. Among other technological applications of QDs, semiconductor memory capacitors, light-emitting diodes (LEDs), and laser diodes should be mentioned.

QDs range in size anywhere from a few lattice parameters to a few micrometers but usually in the nanometer scale. The fluorescence spectrum of larger dots shifts toward red, whereas smaller dots emit bluer light spectrum. QDs, depending on their particular usages, are grown, at large, in pyramidal or spherical geometries having periodic distribution or arbitrary distribution of several dots. Normally, the spherical core-shell QDs are 
chemically synthesized. The pyramidal shapes of the self-assembled QDs grown via Stranski-Krastanov growth mode have been observed by Mo et al. [1] using scanning tunneling microscopy and by Grundmann et al. [2] using high-resolution transmission electron microscopy. Formation of self-organized Ge QWRs on Si(111) substrate with the aid of Stranski-Krastanov growth mode has been reported by Jin et al. [3]. Typical periodic distributions of pyramidal and spherical QDs over a wetting layer of a few atomic layers thick, which have been observed by electron scanning microscopy [4], are shown in Figure 1A and B, respectively. For some applications, as in QD lasers, it is advantageous to use multilayer rather than single-layer distribution of QDs; such a substitution may improve the efficiency of the QS device substantially. Therefore, an analytical formulation for the electro-elastic fields of a three-dimensional (3D) periodic distribution of strongly interacting embedded QDs is of interest; numerous problems may be treated as special cases of this formulation. For example, the electro-elastic fields of a single QD, one or several layers of periodically distributed QDs, two or more interacting QDs, and as such can be mentioned.

The initial misfit strains within the embedded QDs/ QWRs stemming from the lattice mismatch between the QDs/QWRs and the surrounding matrix (barrier) lead to the generation of electro-elastic fields in both the QDs/ QWRs and the barrier due to the intrinsic electro-mechanical interaction exhibited by crystalline materials with no inversion symmetry. The mentioned lattice mismatch serves as the driving force for the formation of the selforganized QDs/QWRs during Stranski-Krastanov growth mode through molecular beam epitaxy (MBE). This intrinsic lattice strain, which changes the interatomic distances and consequently causes the energy levels and the bonding electrons to change, can have a remarkable effect on the electronic and optical properties of semiconductor crystalline QDs/QWRs. Thus, in general, the knowledge of the strain field associated with QSs is useful not only for understanding the creation and evolution of the semiconductor QSs but also is of great value in the design of optoelectronic and microelectronic devices.
To date, there are no analytical models that fully account for the electro-mechanical properties of the QD-/ QWR-barrier system as well as the interaction between the QDs/QWRs. A proper model must (1) treat the QDs/QWRs and the barrier as piezoelectric materials with distinct electro-mechanical properties, (2) properly account for the anisotropic response of the QDs/QWRs and the barrier, and (3) properly incorporate for the electro-mechanical interaction between the QDs/QWRs. The previous analytical models have resorted to some simplifying assumptions. For example, Grundmann et al. [5] studied the strain distribution for InAs/GaAs QD by numerical simulation and also derived analytical results for the simple case of purely elastic isotropic QD in which the piezoelectric coupling has been disregarded. Also, Faux and colleagues [6], based on the simplifying assumption that the QWR and the surrounding barrier are made of the same purely elastic isotropic material, presented an analytical method for the calculation of the pertinent strain field by evaluation of certain contour integrals. Davies [7] accounted for the piezoelectricity of the QD and the barrier but assumed that both media are isotropic and possess identical electro-mechanical properties; Davies addressed the electro-mechanical fields of a single QD using the Lamé displacement potential. Later, by neglecting the piezoelectric effects, Faux and Pearson [8] treated the QD and the barrier as identical anisotropic elastic media and provided an analysis based on an expansion for the strain Green's tensor. Pan [9] formulated the electro-elastic fields around a single anisotropic QD embedded in a barrier with identical electro-mechanical properties using the pertinent Green's function but presented numerical examples for the isotropic case only.

The current work presents an analytical formulation without resort to any of the above-mentioned simplifying assumptions and yet have the capability of treating periodic distributions of strongly interacting QDs/QWRs with arbitrary geometries. Any arbitrary distribution of a finite number of strongly interacting QDs/QWRs as well as a single QD/QWR can be treated as special cases of the current theory. Moreover, the theory is capable of handling the more complicated case in which the QDs/QWRs have

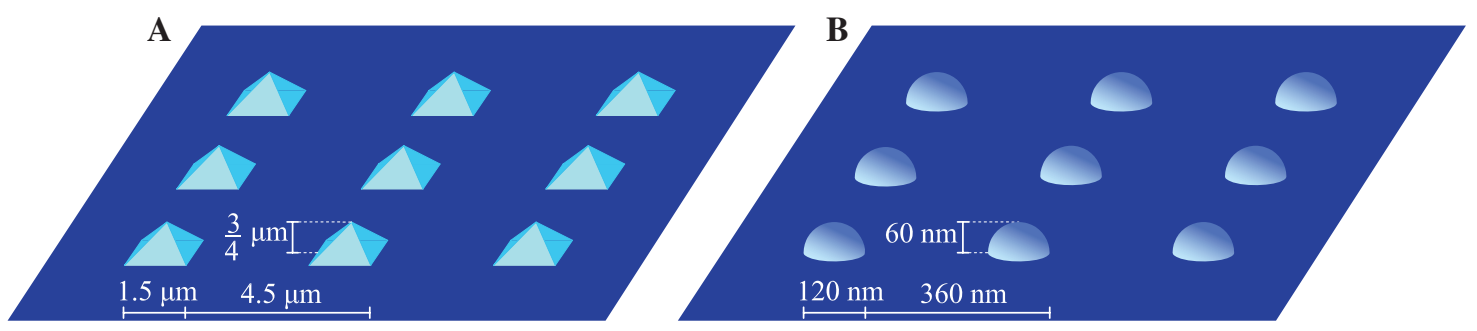

Figure 1 Two typical semiconductors with periodic distributions of (A) pyramidal and (B) spherical QDs. 
multiple coatings - either homogeneous or FG coatings. Another attractive feature of the current analytical formulation is that the misfit strain field can have any arbitrary distribution within the QDs/QWRs and their coatings.

The exact nature of the strain field within an isotropic ellipsoidal inclusion due to the presence of polynomial eigenstrain field inside the same ellipsoidal domain has been given by Eshelby [10]. In the study of the elastic fields inside an anisotropic ellipsoidal inhomogeneity, which is surrounded by an unbounded isotropic matrix under a remote polynomial applied loading, Eshelby [11] simulated the inhomogeneity by an inclusion with a properly chosen eigenstrain field; this approach is well known as the equivalent inclusion method (EIM) in the literature. When the remote applied loading is a polynomial of degree $n \geq 0$, then the proper eigenstrain field inside the equivalent inclusion is also a polynomial of degree $n \geq 0$. It should be emphasized that the mentioned milestone results of Eshelby are bound to a single inhomogeneity with rectilinear anisotropy only. ShokrolahiZadeh and Shodja [12] introduced the Eshelby-Fourier tensor and spectral consistency conditions when studying anisotropic cylindrical multi-inhomogeneities. They showed that presentation of the consistency equations in Fourier space leads to an accurate solution in a rigorous manner for the considered cases. Shodja and Khorshidi [13] addressed the exact nature of the elastic fields of a spherically anisotropic multi-inhomogeneous inclusion and presented the corresponding 3D spectral consistency conditions in the tensor spherical harmonics space. Except for the works of Shokrolahi-Zadeh and Shodja [12] and Shodja and Khorshidi [13], all the other studies, after Eshelby [10, 11], have been customarily developed with respect to the Cartesian coordinates. Consequently, the theoretical developments and results presented in $[12,13]$ are susceptible to misinterpretation by some investigators [14]. For example, Shodja and Khorshidi [13], as one of the rigorous consequences of the derivation of the closed-form expression of the spectral Eshelby tensors within tensor spherical harmonics theories, have stated that the uniform eigenstrain distributions, $\varepsilon_{\theta \theta}^{\star}=\varepsilon_{\phi \phi}^{\star}=1$ as well as $\varepsilon_{r r}^{\star}=1$, within an isotropic inclusion lead to stress and strain fields with logarithmic singularities at the center of the inclusion; an investigator who strictly follows the line of thought and developments of Eshelby [10,11] might strongly disagree with this sound and definitely correct result as did Markenscoff and Dundurs [14]. Markenscoff and Dundurs [14] go on to disprove the mentioned result by referring to a very well-known fact. To quote them, in the first paragraph of the introduction section of their article, they pointed out that "By the very principles of analytic function theory, nite eigenstrain cannot induce stresses and strain fields with singularities, except at points of geometric discontinuities, ...”. Obviously, based on this argument, they expect that the stress and strain fields due to the prescribed uniform eigenstrain fields $\varepsilon_{\theta \theta}^{*}=\varepsilon_{\phi \phi}^{*}=1$ as well as $\varepsilon_{r r}^{*}=1$ within a spherical subdomain must be finite everywhere, including the center of the sphere. Such a wrong conclusion is reached due to lack of attention to the fact that these eigenstrain fields are not defined at the origin, $r=0$; it should be emphasized that, when switching to the accustomed Cartesian coordinates after Eshelby [10, 11], only then will the non-analytic nature of these eigenstrain fields at the origin, $r=0$, unveil.

In the above-mentioned works of Eshelby as well as those of Shodja and colleagues, the matrix is isotropic. In contrast, the present treatment can consider cases where both the piezoelectric matrix and the piezoelectric inhomogeneous inclusion are made of materials with general rectilinear anisotropy in elastic, piezoelectric, and dielectric constants. The current treatment gives a general formulation to account for such a general rectilinear anisotropy. To this end, the 3D electro-mechanical consistency conditions are written in the Fourier space. Within this framework, QWRs/QDs would be considered as inhomogeneous inclusions containing initial misfit strains (eigenstrains).

The current article is organized as follows: Section 2 provides the fundamental equations including the constitutive as well as the governing equations for a piezoelectric medium with general anisotropy containing an arbitrary distribution of electro-elastic inclusions. Subsequently, Section 3 presents an analytical formulation for both cases of periodically and arbitrarily distributed electro-elastic inclusions, where the inclusions can have arbitrary geometries. Section 4 is devoted to the novel electro-mechanical EIM in Fourier space (FEMEIM), which is an important concept toward the determination of the electro-elastic fields due to QWRs/QDs with distinct electro-mechanical properties as compared with the barrier. Several descriptive examples of practical importance are stated, solved, and discussed in Section 5. Section 6 is devoted to the concluding remarks.

\section{Electro-elastic medium with arbitrary distribution of eigen- strain and eigenelectric fields}

Consider an arbitrary distribution of electro-elastic inclusions within a piezoelectric medium with general rectilinear anisotropy in elastic, piezoelectric, and 
dielectric constants. Equivalently, consider a distribution of eigenstrain field, $\boldsymbol{\varepsilon}^{p}$, and a distribution of eigenelectric field, $\mathbf{E}^{p}$, within some subdomains of an infinite piezoelectric material with elastic modulus $\mathbf{C}$, piezoelectric tensor $\mathbf{e}$, and dielectric tensor $\boldsymbol{\kappa}$. The constitutive relations associated to the medium under consideration are given by

$$
\begin{gathered}
\sigma_{i j}=C_{i j k l}\left(\varepsilon_{k l}-\varepsilon_{k l}^{p}\right)-e_{k i j} E_{k}, i, j, k, l=1,2,3, \\
D_{j}=\kappa_{k j}\left(E_{k}-E_{k}^{p}\right)+e_{j k l} \varepsilon_{k l}, j, k, l=1,2,3,
\end{gathered}
$$

where $\boldsymbol{\sigma}$ and $\boldsymbol{\varepsilon}$ are the stress and strain tensors, respectively. $\mathbf{E}$ is the electric field and $\mathbf{D}$ is the electric displacement. The tensors $\mathbf{C}, \boldsymbol{\kappa}$, and $\mathbf{e}$ have the following symmetries:

$$
C_{i j k l}=C_{j i k l}=C_{i j l k}=C_{k l i j}, \quad e_{j k l}=e_{j l k}, \quad \kappa_{k j}=\kappa_{j k}, \quad i, j, k, l=1,2,3 .
$$

In addition, for a displacement field, $\boldsymbol{u}$, and electric potential, $\Phi$, the following relations hold:

$$
\begin{gathered}
\varepsilon_{k l}=\frac{1}{2}\left(u_{k, l}+u_{l, k}\right), \\
E_{k}=-\Phi_{, k} .
\end{gathered}
$$

In the absence of body forces and charges, the equations of equilibrium and charge equation of electrostatics, respectively, become

$$
\begin{aligned}
& \sigma_{i j, j}=0, \\
& D_{j, j}=0 .
\end{aligned}
$$

By virtue of Eqs. (1a), (1b), (3a), (3b), (4a) and (4b), the following differential equations in terms of $\boldsymbol{u}$ and $\Phi$ can be obtained:

$$
\begin{gathered}
C_{i j k l} u_{k, l j}+e_{k i j} \Phi_{, k j}=C_{i j k l} \varepsilon_{k l, j}^{p}, \\
e_{j k l} u_{k, l j}-\kappa_{k j} \Phi_{, k j}=\kappa_{k j} E_{k, j}^{p} .
\end{gathered}
$$

It should be emphasized that Eqs. (5a) and (5b) are valid for the piezoelectric medium with general anisotropy. Because of the intrinsic behavior of piezoelectric materials in converting mechanical to electrical energy and vice versa, the initial misfit strains within the electroelastic inclusions induce elastic and electric fields at both interior and exterior points simultaneously.

\section{Analytical formulation for the electro-elastic fields of peri- odically or arbitrarily distributed electro-elastic inclusions within piezoelectric media}

This section is devoted to the analytical solutions of the system of coupled partial differential Eqs. (5a) and (5b). It is proposed to obtain expressions for the electro-elastic fields due to either periodic or arbitrary distributions of the eigenstrain and eigenelectric fields associated with the unbounded media containing QWRs or QDs. For the case of arbitrary distribution, any finite number of interacting QWRs or QDs with arbitrary geometries as well as arbitrary distance between them may be considered. The formulations are laid down for both general anisotropic and transversely isotropic piezoelectric media. It should be reiterated that the previous analytical works have not differentiated between the electro-mechanical properties of QWRs/QDs and those of the barrier. For this reason, in this section, QWRs/QDs are referred to as electro-elastic inclusions. In Section 4, the electro-mechanical properties will be differentiated, and thus, QWRs/QDs will be treated as electro-elastic inhomogeneous inclusions.

\subsection{Periodic distribution}

Consider a periodic distribution of QWRs or QDs. If the corresponding eigenstrain and eigenelectric fields are quasi differentiable, then they may be expressed in terms of Fourier series as

$$
\begin{aligned}
& \varepsilon_{i j}^{p}(\boldsymbol{x})=\sum_{\boldsymbol{\xi}} \bar{\varepsilon}_{i j}^{p}(\boldsymbol{\xi}) \exp (\mathrm{i} \boldsymbol{\xi} \cdot \boldsymbol{x}), \\
& E_{j}^{p}(\boldsymbol{x})=\sum_{\xi} \bar{E}_{j}^{p}(\xi) \exp (\mathrm{i} \xi \cdot \boldsymbol{x}),
\end{aligned}
$$

where

$$
\bar{\varepsilon}_{i j}^{p}(\boldsymbol{\xi})=\frac{1}{8 L_{1} L_{2} L_{3}} \int_{\Omega} \varepsilon_{i j}^{p}\left(\boldsymbol{x}^{\prime}\right) \exp \left(-\mathrm{i} \xi \cdot \boldsymbol{x}^{\prime}\right) \mathrm{d} \boldsymbol{x}^{\prime},
$$

$$
\bar{\varepsilon}_{i j}^{p}(\xi)=\frac{1}{8 L_{1} L_{2} L_{3}} \int_{\Omega} \varepsilon_{i j}^{p}\left(\boldsymbol{x}^{\prime}\right) \exp \left(-\mathrm{i} \xi \cdot \boldsymbol{x}^{\prime}\right) \mathrm{d} \boldsymbol{x}^{\prime},
$$

$\mathrm{i}=\sqrt{-1}, \boldsymbol{\xi}$ is the wave vector corresponding to the given period of distribution, $\boldsymbol{\Omega}$ is the QD domain in a single period, and $2 L_{1}, 2 L_{2}$, and $2 L_{3}$ are the periods in the $x_{1}^{-}, x_{2}^{-}$, 
and $x_{3}$-directions, respectively. Subsequently, the solutions to the coupled system of Eqs. (5a) and (5b) are taken in the form:

$$
\begin{aligned}
& u_{k}(\boldsymbol{x})=\sum_{\xi} \bar{u}_{k}(\xi) \exp (\mathrm{i} \xi \cdot \boldsymbol{x}), \\
& \Phi(\boldsymbol{x})=\sum_{\xi} \bar{\Phi}(\boldsymbol{\xi}) \exp (\mathrm{i} \xi \cdot \boldsymbol{x})
\end{aligned}
$$

Substitution into Eqs. (5a) and (5b) results in the following system of equations for the unknowns $\bar{u}_{k}$ and $\bar{\Phi}$ :

$$
\begin{gathered}
C_{i j k l} \xi_{l} \xi_{j} \bar{u}_{k}(\xi)+e_{k i j} \xi_{k} \xi_{j} \bar{\Phi}(\xi)=-\mathrm{i} C_{i j k l} \xi_{j} \bar{\varepsilon}_{k l}^{p}(\xi), \\
e_{j k l} \xi_{l} \xi_{j} \bar{u}_{k}(\xi)-\kappa_{k j} \xi_{k} \xi_{j} \bar{\Phi}(\xi)=-\mathrm{i} \kappa_{k j} \xi_{j} \bar{E}_{k}^{p}(\xi) .
\end{gathered}
$$

Introducing

$$
\begin{array}{cc}
K_{i k}(\xi)=K_{k i}(\xi)=C_{i j k l} \xi_{l} \xi_{j}, & i, j, k, l=1,2,3, \\
K_{i 4}(\xi)=K_{4 i}(\xi)=e_{k i j} \xi_{k} \xi_{j}, & i, j, k=1,2,3, \\
K_{44}(\xi)=-\kappa_{k j} \xi_{k} \xi_{j}, & j, k=1,2,3,
\end{array}
$$

$\bar{u}_{k}(\xi)$ and $\bar{\Phi}(\xi)$ are readily obtained by simultaneous solution of Eqs. (9a) and (9b). Subsequently, it can be shown that Eqs. (8a) and ( $8 b)$ reduce to

$$
\begin{aligned}
u_{i}(\boldsymbol{x})= & -\mathrm{i} \sum_{\xi}\left[C_{j k l m} \xi_{k} \bar{\varepsilon}_{l m}^{p}(\xi) N_{i j}(\xi)\right. \\
& \left.+\kappa_{k j} \xi_{j} \bar{E}_{k}^{p}(\xi) N_{i 4}(\xi)\right] D^{-1}(\xi) \exp (\mathrm{i} \xi \cdot \boldsymbol{x}), \\
\Phi(\boldsymbol{x})= & -\mathrm{i} \sum_{\xi}\left[C_{j k l m} \xi_{k} \bar{\varepsilon}_{l m}^{p}(\xi) N_{4 j}(\xi)\right. \\
& \left.+\kappa_{k j} \xi_{j} \bar{E}_{k}^{p}(\xi) N_{44}(\xi)\right] D^{-1}(\xi) \exp (\mathrm{i} \xi \cdot \boldsymbol{x}),
\end{aligned}
$$

where $N_{i j}(\xi)$ and $D(\xi)$ are the cofactors and the determinant of the matrix $\mathbf{K}(\boldsymbol{\xi})$ with components $K_{i j}(\boldsymbol{\xi}), i, j=1,2$, 3, 4. Knowledge of $N_{i j}(\boldsymbol{\xi}), D(\xi), \bar{\varepsilon}_{l m}^{p}(\xi)$, and $\bar{E}_{k}^{p}(\xi)$ for a piezoelectric medium with a specified material anisotropy as well as a given periodic distribution of eigenstrain and eigenelectric fields, which are not necessarily uniform, leads to the calculations of the displacement and electric potential fields using Eqs. (11a) and (11b).

As it will be seen in Sections 3.1.1 and 3.1.2, for a transversely isotropic piezoelectric body with periodic distribution of QWRs and QDs, respectively, the expressions for the electro-mechanical fields simplify considerably.

\subsubsection{QWRs in a transversely isotropic piezoelectric medium}

For a transversely isotropic piezoelectric medium with $x_{3}$ as axis of rotational symmetry as well as axis of polarization, the elastic moduli tensor, $\mathbf{C}$, piezoelectric tensor, $\mathbf{e}$, and dielectric tensor, $\boldsymbol{\kappa}$ are in the forms

$$
\mathbf{C}=\left[\begin{array}{cccccc}
C_{11} & C_{12} & C_{13} & 0 & 0 & 0 \\
C_{12} & C_{11} & C_{13} & 0 & 0 & 0 \\
C_{13} & C_{13} & C_{33} & 0 & 0 & 0 \\
0 & 0 & 0 & C_{44} & 0 & 0 \\
0 & 0 & 0 & 0 & C_{44} & 0 \\
0 & 0 & 0 & 0 & 0 & \frac{C_{11}-C_{12}}{2}
\end{array}\right],
$$

$$
\begin{gathered}
\boldsymbol{\kappa}=\left[\begin{array}{ccc}
\kappa_{11} & 0 & 0 \\
0 & \kappa_{11} & 0 \\
0 & 0 & \kappa_{33}
\end{array}\right], \\
\mathbf{e}=\left[\begin{array}{cccccc}
0 & 0 & 0 & 0 & e_{15} & 0 \\
0 & 0 & 0 & e_{15} & 0 & 0 \\
e_{31} & e_{31} & e_{33} & 0 & 0 & 0
\end{array}\right],
\end{gathered}
$$

respectively.

Theorem 1. For two-dimensional (2D) problem of a periodic distribution of QWRs in a transversely isotropic piezoelectric barrier where the rotational symmetry and polarization axes coincide, the prescribed initial eigenstrain and eigenelectric fields are electrically and elastically impotent, respectively.

Proof. The problem of interest is 2D, and subsequently the expressions for the determinant $D(\xi)$ and the cofactors $N_{i j}(\boldsymbol{\xi})$ reduce to

$$
\begin{aligned}
D\left(\xi_{1}, \xi_{2}, 0\right) & =-\varsigma^{8} \alpha_{1} \alpha_{2} \alpha_{3}, \\
N_{11}\left(\xi_{1}, \xi_{2}, 0\right) & =-\varsigma^{4} \alpha_{3}\left(\alpha_{2} \xi_{1}^{2}+\alpha_{1} \xi_{2}^{2}\right), \\
N_{22}\left(\xi_{1}, \xi_{2}, 0\right) & =-\varsigma^{4} \alpha_{3}\left(\alpha_{1} \xi_{1}^{2}+\alpha_{2} \xi_{2}^{2}\right), \\
N_{33}\left(\xi_{1}, \xi_{2}, 0\right) & =-\kappa_{11} \varsigma^{6} \alpha_{1} \alpha_{2}, \\
N_{12}\left(\xi_{1}, \xi_{2}, 0\right) & =\frac{C_{11}+C_{12}}{2} \varsigma^{4} \alpha_{3} \xi_{1} \xi_{2}, \\
N_{34}\left(\xi_{1}, \xi_{2}, 0\right) & =-e_{15} \varsigma^{6} \alpha_{1} \alpha_{2}, \\
N_{44}\left(\xi_{1}, \xi_{2}, 0\right) & =\varsigma^{6} \alpha_{1} \alpha_{2} \alpha_{4}, \\
N_{13}\left(\xi_{1}, \xi_{2}, 0\right) & =N_{23}\left(\xi_{1}, \xi_{2}, 0\right)=N_{14}\left(\xi_{1}, \xi_{2}, 0\right) \\
& =N_{24}\left(\xi_{1}, \xi_{2}, 0\right)=0,
\end{aligned}
$$

where 


$$
\begin{aligned}
& \varsigma^{2}=\xi_{1}^{2}+\xi_{2}^{2}, \\
& \alpha_{1}=C_{11}, \\
& \alpha_{2}=\frac{C_{11}-C_{12}}{2}, \\
& \alpha_{3}=e_{15}^{2}+C_{44} \kappa_{11}, \\
& \alpha_{4}=C_{44} .
\end{aligned}
$$

Thus, Eqs. (11a) and (11b), which stand for the displacement and electric potential fields, reduce to

$$
\begin{aligned}
u_{i}(\boldsymbol{x})= & -\mathrm{i} \sum_{\xi}\left[C_{j k l m} \xi_{k} \bar{\varepsilon}_{l m}^{p}(\xi) N_{i j}(\xi)\right] D^{-1}(\xi) \exp (\mathrm{i} \xi \cdot \boldsymbol{x}), \\
& i, j, k, l, m=1,2, \\
\Phi(\boldsymbol{x})= & -\mathrm{i} \sum_{\xi}\left[\kappa_{k j} \xi_{j} \bar{E}_{k}^{p}(\xi) N_{44}(\xi)\right] D^{-1}(\xi) \exp (\mathrm{i} \xi \cdot \boldsymbol{x}), j, k=1,2 .
\end{aligned}
$$

Thus, the electrical and the mechanical fields become independent. In other words, the prescribed initial eigenstrain field and eigenelectric field are electrically and elastically impotent, respectively. This is clearly seen from Eqs. (15a) and (15b).

Now, suppose that the QWRs contain dilatational eigenstrain and constant eigenelectric fields:

$$
\begin{gathered}
\varepsilon_{i j}^{p}(\boldsymbol{x})=\delta_{i j} \varepsilon^{p}, i, j=1,2, \\
E_{1}^{p}(\boldsymbol{x})=E_{2}^{p}(\boldsymbol{x})=E^{p},
\end{gathered}
$$

where $\delta_{i j}$ is the Kronecker $\delta$ and $\varepsilon^{p}$ and $E^{p}$ are constants. Thus, for a periodic distribution of QWRs with periods $2 L_{1}$ and $2 L_{2}$ in the $x_{1}$ - and $x_{2}$-directions, respectively, the coefficients in the Fourier representations of the eigenstrain and eigenelectric fields are given by

$$
\begin{aligned}
& \bar{\varepsilon}_{11}^{p}(\xi)=\bar{\varepsilon}_{22}^{p}(\xi)=\bar{\varepsilon}^{p}(\xi)=\frac{\varepsilon^{p}}{4 L_{1} L_{2}} \int_{\Omega} \exp \left(-\mathrm{i} \xi \cdot \boldsymbol{x}^{\prime}\right) \mathrm{d} \boldsymbol{x}^{\prime}, \\
& \bar{E}_{1}^{p}(\xi)=\bar{E}_{2}^{p}(\xi)=\bar{E}^{p}(\xi)=\frac{E^{p}}{4 L_{1} L_{2}} \int_{\Omega} \exp \left(-\mathrm{i} \xi \cdot \boldsymbol{x}^{\prime}\right) \mathrm{d} \boldsymbol{x}^{\prime} .
\end{aligned}
$$

Subsequently, the displacement field $\boldsymbol{u}$ and the electric potential $\Phi$ become

$$
\begin{aligned}
u_{i}(\boldsymbol{x})=-\mathrm{i} \sum_{\boldsymbol{\xi}} \bar{\varepsilon}^{p}(\xi)\left[\left(C_{11}+C_{12}\right) \xi_{1} N_{i 1}(\xi)\right. \\
\left.+\left(C_{11}+C_{12}\right) \xi_{2} N_{i 2}(\xi)\right] D^{-1}(\xi) \exp (\mathrm{i} \xi \cdot \boldsymbol{x}), i=1,2, \\
\Phi(\boldsymbol{x})=-\mathrm{i} \sum_{\xi} \bar{E}^{p}(\xi)\left[\kappa_{11}\left(\xi_{1}+\xi_{2}\right) N_{44}(\xi)\right] D^{-1}(\xi) \exp (\mathrm{i} \xi \cdot \boldsymbol{x})
\end{aligned}
$$

\subsubsection{QDs in a transversely isotropic piezoelectric medium}

Consider a periodic distribution of QDs with an arbitrary geometry throughout a 3D transversely isotropic piezoelectric medium, for which the elastic moduli tensor, C, piezoelectric tensor, e, and dielectric tensor, $\boldsymbol{\kappa}$, are in the form given by Eqs. (12a), (12b), and (12c), respectively. Under these conditions, the expressions for the determinant, $D(\boldsymbol{\xi})$, and components of the cofactor, $\mathbf{N}(\boldsymbol{\xi})$, defined in Section 3.1, can be obtained. The components of $\mathbf{N}(\boldsymbol{\xi})$ are given in Appendix A, and the determinant is as follows:

$$
\begin{aligned}
D\left(\xi_{1}, \xi_{2}, \xi_{3}\right)= & -\frac{1}{2}\left(\left(C_{11}-C_{12}\right)\left(\xi_{1}^{2}+\xi_{2}^{2}\right)+2 C_{44} \xi_{3}^{2}\right)\left[\left(C_{44} e_{31}^{2}-C_{13}^{2} \kappa_{11}\right.\right. \\
& \left.-2 C_{13}\left(e_{15}\left(e_{15}+e_{31}\right)+C_{44} \kappa_{11}\right)\right)\left(\xi_{1}^{2}+\xi_{2}^{2}\right)^{2} \xi_{3}^{2} \\
& +\left(-2\left(C_{44} e_{31}+C_{13}\left(e_{15}+e_{31}\right)\right) e_{33}\right. \\
& +C_{33}\left(\left(e_{15}+e_{31}\right)^{2}+C_{44} \kappa_{11}\right) \\
& \left.-C_{13}\left(C_{13}+2 C_{44}\right) \kappa_{33}\right)\left(\xi_{1}^{2}+\xi_{2}^{2}\right) \xi_{3}^{4} \\
& +C_{44}\left(e_{33}^{2}+C_{33} \kappa_{33}\right) \xi_{3}^{6} \\
& +C_{11}\left(\xi_{1}^{2}+\xi_{2}^{2}\right)\left(\left(e_{15}^{2}+C_{44} \kappa_{11}\right)\left(\xi_{1}^{2}+\xi_{2}^{2}\right)^{2}\right. \\
& +\left(2 e_{15} e_{33}+C_{33} \kappa_{11}+C_{44} \kappa_{33}\right)\left(\xi_{1}^{2}+\xi_{2}^{2}\right) \xi_{3}^{2} \\
& \left.\left.+\left(e_{33}^{2}+C_{33} \kappa_{33}\right) \xi_{3}^{4}\right)\right] .
\end{aligned}
$$

Substitution of the expressions for $D(\boldsymbol{\xi})$ and $N_{i j}(\boldsymbol{\xi})$ as well as the expressions for $\bar{\varepsilon}_{l m}^{p}(\xi)$ and $\bar{E}_{k}^{p}(\xi)$ from Eqs. (7a) and (7b) into Eqs. (11a) and (11b) results in the displacement field, $\boldsymbol{u}$, and the electric potential, $\Phi$. In contrast to the 2D case of periodic distribution of QWRs in a transversely isotropic piezoelectric medium, where the electrical and mechanical fields are decoupled, for the present case of QDs, these fields are strongly coupled.

\subsection{Arbitrary distribution}

For an arbitrary distribution of a finite number of QWRs or QDs, the eigenstrain and eigenelectric fields are represented by the Fourier integrals:

$$
\varepsilon_{i j}^{p}(\boldsymbol{x})=\int_{-\infty}^{\infty} \bar{\varepsilon}_{i j}^{p}(\boldsymbol{\xi}) \exp (\mathrm{i} \boldsymbol{\xi} \cdot \boldsymbol{x}) \mathrm{d} \boldsymbol{\xi},
$$

$$
E_{j}^{p}(\boldsymbol{x})=\int_{-\infty}^{\infty} \bar{E}_{j}^{p}(\boldsymbol{\xi}) \exp (\mathrm{i} \boldsymbol{\xi} \cdot \boldsymbol{x}) \mathrm{d} \boldsymbol{\xi},
$$

where

$$
\bar{\varepsilon}_{i j}^{p}(\boldsymbol{\xi})=\frac{1}{(2 \pi)^{3}} \int_{-\infty}^{\infty} \varepsilon_{i j}^{p}\left(\boldsymbol{x}^{\prime}\right) \exp \left(-\mathrm{i} \boldsymbol{\xi} \cdot \boldsymbol{x}^{\prime}\right) \mathrm{d} \boldsymbol{x}^{\prime},
$$




$$
\bar{E}_{j}^{p}(\boldsymbol{\xi})=\frac{1}{(2 \pi)^{3}} \int_{-\infty}^{\infty} E_{j}^{p}\left(\boldsymbol{x}^{\prime}\right) \exp \left(-\mathrm{i} \boldsymbol{\xi} \cdot \boldsymbol{x}^{\prime}\right) \mathrm{d} \boldsymbol{x}^{\prime}
$$

Likewise, if $\bar{u}_{k}$ and $\bar{\Phi}$ are the Fourier transforms of the displacement and electric potential fields, respectively, then

$$
\begin{aligned}
& u_{k}(\boldsymbol{x})=\int_{-\infty}^{\infty} \bar{u}_{k}(\boldsymbol{\xi}) \exp (\mathrm{i} \boldsymbol{\xi} \cdot \boldsymbol{x}) \mathrm{d} \boldsymbol{\xi}, \\
& \Phi(\boldsymbol{x})=\int_{-\infty}^{\infty} \bar{\Phi}(\boldsymbol{\xi}) \exp (\mathrm{i} \boldsymbol{\xi} \cdot \boldsymbol{x}) \mathrm{d} \boldsymbol{\xi} .
\end{aligned}
$$

By applying Fourier transform to Eqs. (5a) and (5b), after some manipulations, it can be shown that

$$
\begin{aligned}
u_{i}(\mathbf{x})= & -\mathrm{i} \int_{-\infty}^{\infty}\left[C_{j k k m} \xi_{k} \bar{\varepsilon}_{l m}^{p}(\xi) N_{i j}(\xi)\right. \\
& \left.+\kappa_{k j} \xi_{j} \bar{E}_{k}^{p}(\xi) N_{i 4}(\xi)\right] D^{-1}(\xi) \exp (\mathrm{i} \xi \cdot \boldsymbol{x}) \mathrm{d} \xi, \\
\Phi(\boldsymbol{x})= & -\mathrm{i} \int_{-\infty}^{\infty}\left[C_{j k k m} \xi_{k} \bar{\varepsilon}_{l m}^{p}(\xi) N_{4 j}(\xi)\right. \\
& \left.+\kappa_{k j} \xi_{j} \bar{E}_{k}^{p}(\xi) N_{44}(\xi)\right] D^{-1}(\xi) \exp (\mathrm{i} \xi \cdot \boldsymbol{x}) \mathrm{d} \xi .
\end{aligned}
$$

The above formulations, which presume nothing about the elastic moduli, piezoelectric, and dielectric tensors, are valid for general electro-mechanical properties. The coupling between the electrical and mechanical fields is exhibited by these expressions as well.

Additionally, for 2D and 3D problems of arbitrary distribution of QWRs and QDs in a transversely isotropic piezoelectric medium with respect to the $x_{3}$-axis, the solutions obtained in Sections 3.1.1 and 3.1.2, respectively, will be representable in terms of the Fourier integral. Therefore, as in the case of periodic distribution of QWRs in such a medium, for the arbitrary distribution, the initial eigenstrain field and eigenelectric field would be electrically and elastically impotent, respectively.

It should be noted that the formulations of this section as well as those of Section 3.1 remain applicable in the case of strong interaction, i.e., high concentration of QWRs or QDs.

\section{Electro-mechanical EIM for QWRs/QDs with FG transition zone in Fourier space}

The available works on the subject, for simplicity, have at large assumed identical elastic and electrical properties for the QWRs/QDs and those of the barrier. In the literature, lattice mismatch between the QWRs/QDs and the barrier has been accounted for through consideration of a misfit strain field, whereas neglecting the distinction between their electro-mechanical properties. Such a simplifying assumption is physically unrealistic and can lead to remarkable inaccuracies in the calculated electro-elastic fields. As will be shown, one of the strengths of the current theory is its capability to properly account for the true electro-mechanical properties of each phase with a little effort. Along this line of thought, a physically more interesting case in which an FG interfacial zone is inserted between the QWRs/QDs and the barrier to remove the interfacial defects and enhance the charge transfer processes is realized. This section gives a remedy to treat problems with such complexities by extending the traditional Eshelby's EIM in polynomial space [11] to the FEMEIM. As it will be seen in this section, the new mathematical formulations in Fourier space can provide a rather general treatise for a broad range of pertinent problems taking the interaction between the inhomogeneous inclusions (QWRs/QDs) and FG transition zones as well as the electro-mechanical coupling into account exactly.

Consider a medium consisting of a periodic distribution of piezoelectric QWRs/QDs with FG transition layer embedded in a piezoelectric barrier; the mentioned phases are made of different piezoelectric materials with distinct electro-mechanical properties. For the time being, assume that there are no misfit strains within the QWRs/QDs as well as within the FG coatings. To treat this problem, the QWRs/QDs and their FG transition layers are equivalently replaced with islands occupying the same space and having the same electro-mechanical properties as those of the barrier but containing eigenstrain field, $\boldsymbol{\varepsilon}^{*}(\boldsymbol{x})$, and eigenelectric field, $\boldsymbol{E}^{*}(\boldsymbol{x})$, such that the stress and electric displacement fields of the original problem are identically equal to those of the homogenized system. If within the QWRs/QDs and their FG coatings a misfit strain field, $\boldsymbol{\varepsilon}^{p}(\boldsymbol{x})$ is initially present, then $\boldsymbol{\varepsilon}^{*}(\boldsymbol{x})$ within the islands of the homogenized system is simply replaced with $\boldsymbol{\varepsilon}^{*}(\boldsymbol{x})+\boldsymbol{\varepsilon}^{p}(\boldsymbol{x})$. It should be emphasized that the homogenizing eigenstrain and eigenelectric fields account for both the inter-inhomogeneity and intra-inhomogeneity electro-mechanical interactions exactly; the inhomogeneity consists of all the phases of QWRs/QDs and their surrounding FG coatings. Next, by utilizing Eqs. (11a) and (11b), the disturbance strain field, $\boldsymbol{\varepsilon}^{d}(\boldsymbol{x})$, and the disturbance electric field, $\boldsymbol{E}^{d}(\boldsymbol{x})$, due to the periodic distribution of the eigenstrain field, $\boldsymbol{\varepsilon}^{*}(\boldsymbol{x})+\boldsymbol{\varepsilon}^{p}(\boldsymbol{x})$, and eigenelectric field, $\boldsymbol{E}^{\star}(\boldsymbol{x})$, in the equivalent homogenized problem can be obtained as 


$$
\begin{aligned}
\varepsilon_{i j}^{d}(\boldsymbol{x})= & \sum_{\xi} \bar{\varepsilon}_{i j}^{d}(\xi) \exp (\mathrm{i} \boldsymbol{\xi} \cdot \boldsymbol{x}) \\
= & \frac{1}{2} \sum_{\xi}\left[C_{n k l m} \xi_{k} \xi_{j}\left(\bar{\varepsilon}_{l m}^{*}(\xi)+\bar{\varepsilon}_{l m}^{p}(\xi)\right) N_{i n}(\xi)\right. \\
& +\kappa_{k n} \xi_{n} \xi_{j} \bar{E}_{k}^{*}(\xi) N_{i 4}(\xi) \\
& +C_{n k l m} \xi_{k} \xi_{i}\left(\bar{\varepsilon}_{l m}^{*}(\xi)+\bar{\varepsilon}_{l m}^{p}(\xi)\right) N_{j n}(\xi) \\
& \left.+\kappa_{k n} \xi_{n} \xi_{i} \bar{E}_{k}^{*}(\xi) N_{j 4}(\xi)\right] D^{-1}(\xi) \exp (\mathrm{i} \xi \cdot \boldsymbol{x}), \\
E_{j}^{d}(\boldsymbol{x})= & \sum_{\xi} \bar{E}_{j}^{d}(\xi) \exp (\mathrm{i} \xi \cdot \boldsymbol{x}) \\
= & -\sum_{\xi}\left[C_{n k l m} \xi_{k} \xi_{j}\left(\bar{\varepsilon}_{l m}^{*}(\xi)+\bar{\varepsilon}_{l m}^{p}(\xi)\right) N_{4 n}(\xi)\right. \\
& \left.+\kappa_{k n} \xi_{n} \xi_{j} \bar{E}_{k}^{*}(\xi) N_{44}(\xi)\right] D^{-1}(\xi) \exp (\mathrm{i} \xi \cdot \boldsymbol{x}),
\end{aligned}
$$

in which $\bar{\varepsilon}_{l m}^{p}(\xi)$ are the components of the Fourier coefficients pertinent to the initial misfit strain field within each of the FG coated QWR/QD ensembles and $\bar{\varepsilon}_{l m}^{*}(\xi)$ and $\bar{E}_{k}^{*}(\xi)$ are those of the homogenizing eigenstrain and eigenelectric fields, respectively. It is convenient to define the elastic moduli tensor, $\hat{\boldsymbol{C}}(\boldsymbol{x})$ associated with the original inhomogeneous inclusion problem as

$$
\hat{\boldsymbol{C}}(\mathbf{x})=\left\{\begin{array}{c}
\boldsymbol{C}^{Q} \\
\boldsymbol{C}^{C}(\boldsymbol{x}), \\
\boldsymbol{C}
\end{array}\right.
$$

where $\boldsymbol{C}^{Q}, \boldsymbol{C}^{C}(\boldsymbol{x})$, and $\boldsymbol{C}$ are the elastic moduli tensors for the QWRs/QDs, coating, and barrier, respectively; the theory does not limit $\boldsymbol{C}^{Q}$ to be constant. The piezoelectric tensor, $\hat{\boldsymbol{e}}(\boldsymbol{x})$ and the dielectric tensor, $\hat{\boldsymbol{\kappa}}(\boldsymbol{x})$ for the inhomogeneous inclusion problem are defined in a similar manner as $\hat{\boldsymbol{C}}(\boldsymbol{x})$. Then, the equivalency of the original inhomogeneous inclusion and the equivalent electromechanical inclusion problems at any field point, $\boldsymbol{x}$ leads to the following consistency conditions:

$$
\begin{aligned}
& \hat{C}_{m n i j}(\boldsymbol{x})\left(\varepsilon_{i j}^{d}(\boldsymbol{x})-\varepsilon_{i j}^{p}(\boldsymbol{x})\right)-\hat{e}_{j m n}(\boldsymbol{x}) E_{j}^{d}(\boldsymbol{x}) \\
& \quad=C_{m n i j}\left(\varepsilon_{i j}^{d}(\boldsymbol{x})-\varepsilon_{i j}^{p}(\boldsymbol{x})-\varepsilon_{i j}^{*}(\boldsymbol{x})\right)-e_{j m n} E_{j}^{d}(\boldsymbol{x}), \\
& \hat{\kappa}_{j m}(\boldsymbol{x}) E_{j}^{d}(\boldsymbol{x})+\hat{e}_{m i j}(\boldsymbol{x}) \varepsilon_{i j}^{d}(\boldsymbol{x})=\kappa_{j m}\left(E_{j}^{d}(\boldsymbol{x})-E_{j}^{*}(\boldsymbol{x})\right)+e_{m i j} \varepsilon_{i j}^{d}(\boldsymbol{x}) .
\end{aligned}
$$

Eqs. (26a) and (26b) correspond to the equivalency of the stress and the electric displacement fields, respectively. Define

$$
\begin{aligned}
\Delta C(x)=\hat{C}(x)-C, \\
\Delta e(x)=\hat{e}(x)-e, \\
\Delta \kappa(x)=\hat{\kappa}(x)-\kappa .
\end{aligned}
$$

Next, using the Fourier series expansions of the electro-mechanical properties and fields, the consistency conditions (26a) and (26b) can be rewritten as

$$
\begin{aligned}
& \sum_{\xi} \sum_{\eta}\left[\overline{\Delta C}_{m n i j}(\eta)\left(\bar{\varepsilon}_{i j}^{d}(\xi)-\bar{\varepsilon}_{i j}^{p}(\xi)\right)\right. \\
& \left.-\overline{\Delta e}_{j m n}(\boldsymbol{\eta}) \bar{E}_{j}^{d}(\boldsymbol{\xi})\right] \exp (\mathrm{i}(\boldsymbol{\xi}+\boldsymbol{\eta}) \cdot \boldsymbol{x}) \\
& =-\sum_{\xi} C_{m n i j} \bar{\varepsilon}_{i j}^{*}(\xi) \exp (\mathrm{i} \xi \cdot \boldsymbol{x}) \text {, } \\
& \sum_{\xi} \sum_{\eta}\left[\overline{\Delta \kappa}_{j m}(\eta) \bar{E}_{j}^{d}(\xi)+\overline{\Delta e}_{m i j}(\eta) \bar{\varepsilon}_{i j}^{d}(\boldsymbol{\xi})\right] \exp (\mathrm{i}(\boldsymbol{\xi}+\boldsymbol{\eta}) \cdot \boldsymbol{x}) \\
& =-\sum_{\boldsymbol{\xi}} \kappa_{j m} \bar{E}_{j}^{*}(\boldsymbol{\xi}) \exp (\mathrm{i} \boldsymbol{\xi} \cdot \boldsymbol{x}),
\end{aligned}
$$

in which $\overline{\Delta C}_{m n i j}(\eta), \overline{\Delta e}_{j m n}(\boldsymbol{\eta})$, and $\overline{\Delta \kappa}_{j m}(\boldsymbol{\eta})$ are the Fourier coefficients corresponding to $\Delta \boldsymbol{C}(\boldsymbol{x}), \Delta \boldsymbol{e}(\boldsymbol{x})$, and $\Delta \boldsymbol{\kappa}(\boldsymbol{x})$, respectively. Substitution of the Fourier coefficients $\bar{\varepsilon}_{i j}^{d}(\xi)$ and $\bar{E}_{j}^{d}(\xi)$ from Eqs. (24a) and (24b) into Eqs. (28a) and (28b) leads to a system of simultaneous equations for the unknowns $\bar{\varepsilon}_{l m}^{*}(\xi)$ and $\bar{E}_{k}^{*}(\xi)$; thus, the Fourier coefficients of the homogenizing eigenstrain and eigenelectric fields are readily available, and subsequently, the disturbance strain and disturbance electric fields can be evaluated from Eqs. (24a) and (24b), respectively.

For an arbitrary distribution, $\mathfrak{D}_{a}$, of a finite number of interacting FG-coated QWRs/QDs, the Fourier series in the consistency conditions (28a) and (28b) must be replaced with Fourier integrals accordingly, as discussed in Section 3.2. Alternatively, one may come up with an accurate solution by working with the Fourier series, where distribution $\mathfrak{D}_{a}$ is taken as a unit cell that is distributed periodically throughout the entire medium with a large enough period such that the interaction between the unit cells is negligibly small.

Theorem 2. For $2 D$ problems of either a periodic or an arbitrary distribution of FG coated inhomogeneous QWRs in a transversely isotropic piezoelectric barrier where the axes of the rotational symmetry and polarization coincide, the initial misfit strain, $\boldsymbol{\varepsilon}^{p}(\boldsymbol{x})$ is electrically impotent.

Proof. For plane electro-mechanical problems for which the axes of the rotational symmetry and polarization of the piezoelectric medium coincide with the $x_{3}$-axis, the 1,2 components of the piezoelectric tensor are identically equal to zero, i.e., $\hat{e}_{j m n}=e_{j m n}=0, j, m, n=1,2$. Thus, the inplane components of the stress and electric displacement fields given by Eqs. (1a) and (1b) are independent of the inplane components of the electric and strain fields, respectively. Subsequently, the consistency conditions (28a) and (28b) will reduce to 


$$
\begin{aligned}
& \sum_{\boldsymbol{\xi}} \sum_{\boldsymbol{\eta}} \overline{\Delta C}_{m n i j}(\boldsymbol{\eta})\left(\bar{\varepsilon}_{i j}^{d}(\boldsymbol{\xi})-\bar{\varepsilon}_{i j}^{p}(\boldsymbol{\xi})\right) \exp (\mathrm{i}(\boldsymbol{\xi}+\boldsymbol{\eta}) \cdot \boldsymbol{x}) \\
& =-\sum_{\xi} C_{m n i j} \bar{\varepsilon}_{i j}^{*}(\boldsymbol{\xi}) \exp (\mathrm{i} \xi \cdot \boldsymbol{x}), i, j, m, n=1,2, \\
& \sum_{\boldsymbol{\xi}} \sum_{\boldsymbol{\eta}} \overline{\Delta \kappa}_{j m}(\boldsymbol{\eta}) \bar{E}_{j}^{d}(\boldsymbol{\xi}) \exp (\mathrm{i}(\boldsymbol{\xi}+\boldsymbol{\eta}) \cdot \boldsymbol{x}) \\
& =-\sum_{\xi} \kappa_{j m} \bar{E}_{j}^{*}(\xi) \exp (\mathrm{i} \xi \cdot \boldsymbol{x}), \quad j, k, m=1,2 .
\end{aligned}
$$

In addition, according to Theorem 1, both the misfit and homogenizing eigenstrain fields do not disturb the electric fields. Conversely, the homogenizing eigenelectric field does not affect the elastic fields. Therefore, from Eq. (24b)

$$
\bar{E}_{j}^{d}(\xi)=-\kappa_{k n} \xi_{n} \xi_{j} N_{44}(\xi) D^{-1}(\xi) \bar{E}_{k}^{*}(\xi) .
$$

It can be clearly seen that the mechanical and electrical consistency conditions, respectively, given by Eqs. (29a) and (29b) become fully decoupled. Substituting Eq. (30) into Eq. (29b) immediately results in

$$
\bar{E}_{1}^{\star}(\xi)=\bar{E}_{2}^{\star}(\xi)=0 \text {. }
$$

This implies that $\bar{E}_{j}^{d}(\xi) \equiv 0$, and consequently, no electric field would be induced in the medium.

Remark 1. Under similar conditions described in Theorem 2, an arbitrary initial eigenelectric field, $\mathbf{E}^{p}(\boldsymbol{x})$, within the QWRs is mechanically impotent.

Corollary 1. Under the conditions of Theorem 2, the total strain and total electric fields for a periodic distribution of QWRs become

$$
\begin{aligned}
\varepsilon_{i j}^{d}(\boldsymbol{x})= & \frac{1}{2} \sum_{\xi}\left[C_{n k l m} \xi_{k} \xi_{j}\left(\bar{\varepsilon}_{l m}^{*}(\boldsymbol{\xi})+\bar{\varepsilon}_{l m}^{p}(\xi)\right) N_{i n}(\boldsymbol{\xi})\right. \\
& \left.+C_{n k l m} \xi_{k} \xi_{i}\left(\bar{\varepsilon}_{l m}^{*}(\xi)+\bar{\varepsilon}_{l m}^{p}(\xi)\right) N_{j n}(\xi)\right] D^{-1}(\xi) \exp (\mathrm{i} \xi \cdot \boldsymbol{x}), \\
& i, j=1,2,
\end{aligned}
$$

$$
E_{j}^{d}(\boldsymbol{x})=0, j=1,2,
$$

in which $N_{i j}(\boldsymbol{\xi})$ and $D(\boldsymbol{\xi})$ are given by Eq. (13), $\bar{\varepsilon}_{l m}^{p}(\boldsymbol{\xi})$ is given by Eq. (7a), and $\bar{\varepsilon}_{l m}^{*}(\xi)$ are obtained from consistency condition (29a) given in Theorem 2.

Proof. The statement of this corollary is readily verified as a consequence of Theorem 2 in conjunction with Eqs. (24a) and (24b).

\section{Numerical examples}

For a demonstration, different examples of periodic and nonperiodic distributions of QDs and QWRs at high concentration are considered; the presented analytical formulations can result in highly accurate results even for the high concentration cases where the QWRs or QDs are strongly interacting. Moreover, different shapes and orientations of QWRs or QDs, which are of particular importance in science and engineering, are considered.

For the sake of verification, the first example is devoted to the simple problem of an isotropic spherical inclusion (single isotropic spherical QD), which has been previously considered in the literature. The second example addresses the elastic and electric fields due to a periodic distribution of QWRs with quadrangular cross section, whereas the third example deals with a periodic distribution of pyramidal QDs. In the fourth example, the disturbance in the electro-elastic fields of a single pyramidal QD by a nearby (strongly interacting) pyramidal QD is studied and discussed. Examples 2-4 are all pertinent to the transversely isotropic piezoelectric barrier made of AlN and transversely isotropic piezoelectric QWRs and QDs made of InN; the elastic and electrical properties of the considered piezoelectric materials are given in Table $1[15,16]$.

\subsection{Single spherical QD}

Previously, Pan [9] has modeled a single spherical QD with radius of 1 unit within an AlN barrier as an inclusion problem with dilatational eigenstrain field. More strictly speaking, Pan [9] considered an unbounded homogeneous piezoelectric medium made of AlN within which a spherical region of radius 1 unit contains a dilatational eigenstrain field, $\varepsilon_{i j}^{p}$ :

$$
\varepsilon_{i j}^{p}=0.07 \delta_{i j} .
$$

Moreover, Pan [9] assumed that the medium is isotropic. In the context of the current theory, the exact expression

Table 1 Elastic constants (in $\mathrm{GPa}$ ), piezoelectric constants (in $\mathrm{C} / \mathrm{m}^{2}$ ), and dielectric constants (in F/m) for AIN and InN.

\begin{tabular}{lllllllllll}
\hline Material & $C_{11}$ & $C_{12}$ & $C_{13}$ & $C_{33}$ & $C_{44}$ & $e_{31}$ & $e_{33}$ & $e_{15}$ & $\kappa_{11}$ & $\kappa_{33}$ \\
\hline AIN & 396 & 137 & 108 & 373 & 116 & -0.58 & 1.55 & -0.48 & $9 \kappa_{0}$ & $11 \kappa_{0}$ \\
InN & 223 & 115 & 92 & 224 & 48 & -0.57 & 0.97 & -0.22 & $15.3 \kappa_{0}$ & $15.3 \kappa_{0}$ \\
\hline
\end{tabular}

$\kappa_{0}$ is the dielectric constant for vacuum, $\kappa_{0}=8.854 \times 10^{-12}$. 
for the corresponding Fourier coefficient, $\bar{\varepsilon}_{i j}^{p}$ is obtained as

$$
\bar{\varepsilon}_{i j}^{p}=\left(\frac{1}{2 \pi}\right)^{3} \frac{(2 \pi)^{3 / 2} J_{3 / 2}\left(\left(\xi_{1}^{2}+\xi_{2}^{2}+\xi_{3}^{2}\right)^{1 / 2}\right)}{\left(\xi_{1}^{2}+\xi_{2}^{2}+\xi_{3}^{2}\right)^{3 / 4}} \varepsilon_{i j}^{p} .
$$

For demonstration, the variation of the $x_{1}$-component of the electric field, $E_{1}$ along the line $-2.5 \leq x_{1}=x_{2}=x_{3} \leq 2.5$ is presented in Figure 2. As it is observed, the jumps in the strain component, $\varepsilon_{11}$, and in the electric field component, $E_{1}$, across the QD-barrier interface are captured. The electric field component, $E_{1}$, is vanishingly small within the QD. Meanwhile, the corresponding plot in [9], as shown in Figure 2, has been provided over the interval $1 \leq x_{1}=x_{2}=x_{3} \leq 6$, which is pertinent to only the exterior points of the QD; moreover, the plot is not extended to the close vicinity of the point $x_{1}=x_{2}=x_{3} \approx 0.577$ located on the QD-matrix interface. It should be noted that the methodology used in [9] is based on obtaining the Green's function for the problem by utilization of the Stroh formalism.

\subsection{Periodic distribution of quadrangular $\operatorname{InN}$ QWRs within AIN barrier}

In contrast to the previous example, in this example, QWRs are modeled as inhomogeneous inclusions rather than just inclusions. More strictly speaking, the realistically distinct electro-mechanical properties of the InN QWRs from those of the AlN barrier is taken into account properly. The transversely isotropic nature of the QWRs and the barrier are also taken into consideration. The electro-mechanical properties of InN and AlN are provided in Table 1. Suppose the axes of the rotational symmetry and polarization of both the QWRs and the barrier coincide with the $x_{3}$-axis. According to Theorem 2 given in Section 4,

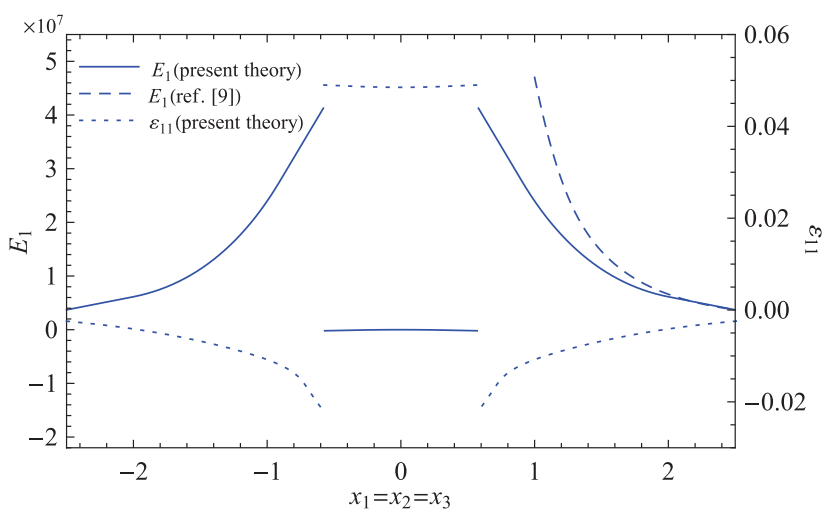

Figure 2 Variations of the component of the total electric field, $E_{1}$, and total strain, $\varepsilon_{11}$, along the line $x_{1}=x_{2}=x_{3}$. the corresponding initial eigenstrain and eigenelectric field are electrically and mechanically impotent, respectively, and the total strain and total electric fields are given by Eqs. (31a) and (31b) of Corollary 1, respectively. Assume that the QWRs are distributed with periodicity of 2 units in the $2 \mathrm{D}$ space and their dimensions are $2 / 3 \times 2 / 3$ as shown in Figure 3.

Consider the following dilatational eigenstrain field within the QWRs:

$$
\varepsilon_{i j}^{p}=\delta_{i j}, \quad i, j=1,2 .
$$

The exact expression for the corresponding Fourier coefficient, $\bar{\varepsilon}_{i j}^{p}(\xi)$ becomes

$$
\bar{\varepsilon}_{i j}^{p}(\xi)=\frac{1}{\xi_{1} \xi_{2}} \sin \left(\xi_{1} / 3\right) \sin \left(\xi_{2} / 3\right) \delta_{i j}, \quad i, j=1,2 .
$$

For a demonstration, the variations of the components of the total strain $\varepsilon_{11}$ with $x_{1}\left(\left|x_{1}\right| \leq 1\right)$ along $x_{2}=0$ and $x_{2}=1$ are plotted in Figure 4 (Case 1). To examine the effect of the assumption of identical electro-mechanical properties for both the QWRs and the barrier, let both regions be made of AlN; this results in the distributions indicated as Case 2 in Figure 4. Comparison of the results of Cases 1 and 2 reveals a notable difference, as large as $\sim 8 \%$ where the local maximum and local minimum values of $\varepsilon_{11}$ occur.

\subsection{D periodic distribution of pyramidal InN QDs in AlN barrier}

The formation of self-organized QDs into vertically oriented pyramidal shape has been widely observed through

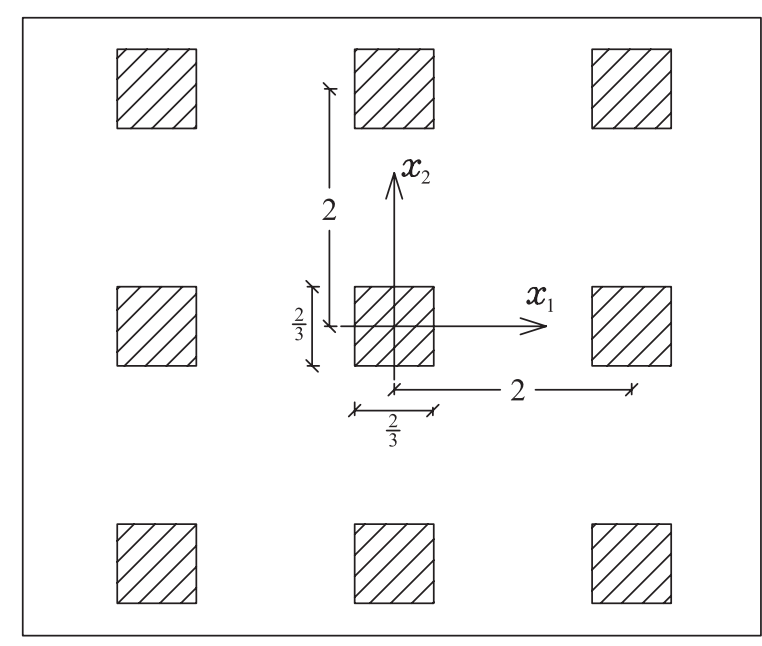

Figure 3 Periodic distribution of quadrangular InN QWRs within AIN barrier in the $x_{1} x_{2}$-plane. 


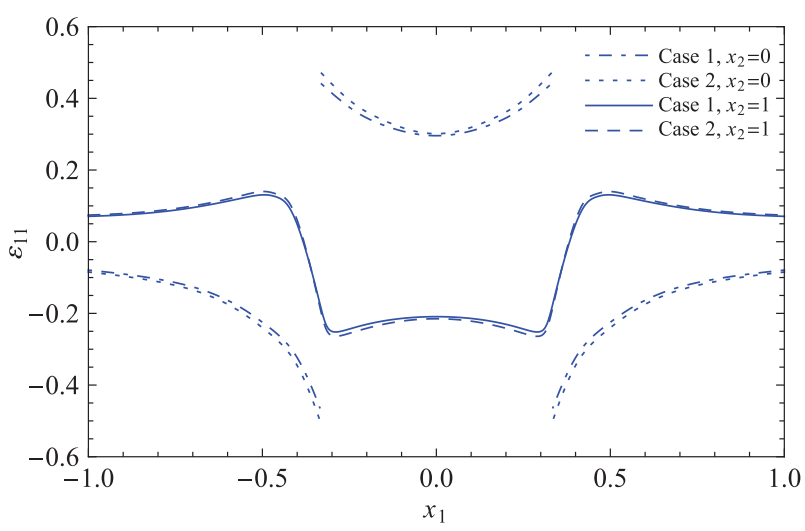

Figure 4 Variation of the component of the total strain $\varepsilon_{11}$ as a function of $x_{1}$ along $x_{2}=0$ and $x_{2}=1$.

high-resolution transmission electron microscopy. In this example, the 3D periodic distribution of pyramidal InN QDs in an AlN barrier will be studied. The axes of the rotational symmetry and polarization of both the QDs and the barrier coincide with the $x_{3}$-axis. The vertical axis of the pyramidal QDs is parallel to the $x_{3}$-direction and their quadrangular cross section is parallel to the $x_{1} x_{2}$-plane as shown in Figure 5. Assume that the QDs are distributed with periodicity of 2 units in the $x_{i}$-direction, $i=1,2,3$. The dimensions of the base and the height of the pyramids are assumed to be equal to $2 / 3 \times 2 / 3$ and $1 / 3$, respectively.

Suppose that

$$
\varepsilon_{i j}^{p}=\delta_{i j} \text {, }
$$

is the prescribed dilatational eigenstrain field within each of the QDs. Then the expression of the corresponding

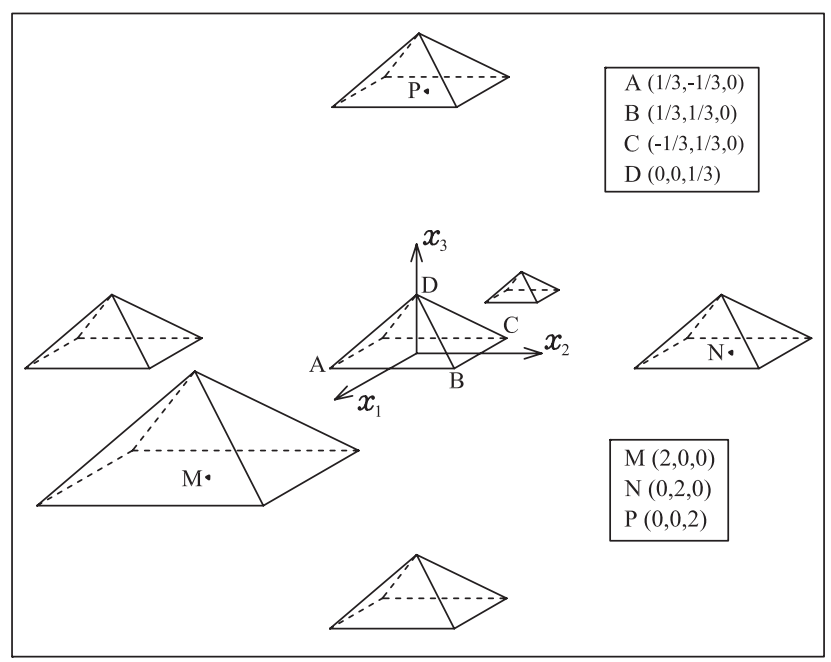

Figure 5 3D periodic distribution of pyramidal InN QDs within AIN barrier.
Fourier coefficient, $\bar{\varepsilon}_{i j}^{p}(\xi)$ can be calculated exactly using Eq. (7a):

$$
\begin{aligned}
& \varepsilon_{i j}^{-p}(\xi)=\frac{1}{4 \xi_{1} \xi_{2}}\left[-\frac{4 i e^{-\frac{i \xi_{3}}{3}} \xi_{1} \xi_{2} \xi_{3}}{\xi_{1}^{4}+\left(\xi_{2}^{2}-\xi_{3}^{2}\right)^{2}-2 \xi_{1}^{2}\left(\xi_{2}^{2}+\xi_{3}^{2}\right)}\right. \\
& +\frac{i \xi_{3} \operatorname{Cos}\left[\frac{1}{3}\left(-\xi_{1}+\xi_{2}\right)\right]+\left(\xi_{1}-\xi_{2}\right) \operatorname{Sin}\left[\frac{\xi_{1}-\xi_{2}}{3}\right]}{\left(\xi_{1}-\xi_{2}\right)^{2}-\xi_{3}^{2}} \\
& \left.-\frac{i \xi_{3} \operatorname{Cos}\left[\frac{\xi_{1}+\xi_{2}}{3}\right]+\left(\xi_{1}+\xi_{2}\right) \operatorname{Sin}\left[\frac{\xi_{1}+\xi_{2}}{3}\right]}{\left(\xi_{1}+\xi_{2}-\xi_{3}\right)\left(\xi_{1}+\xi_{2}+\xi_{3}\right)}\right] \delta_{i j} \text {. }
\end{aligned}
$$

The variations of $\varepsilon_{11}$ and $\sigma_{11}$ with $x_{1}\left(\left|x_{1}\right| \leq 1\right)$ in $x_{3}=0$ plane along $x_{2}=0$ ( $x_{1}$-axis) and along $x_{2}=1$ are shown in Figure 6. Along $x_{2}=0$, while the stress component $\sigma_{11}$ is continuous across the QD-barrier interface $( \pm 1 / 3,0,0)$, the strain component $\varepsilon_{11}$ has a jump there. Meanwhile, both $\varepsilon_{11}$ and $\sigma_{11}$ along the line $x_{2}=1$ (midway between two QDs), which entirely lies within the barrier are continuous everywhere. However, as seen from Figure 6, their variations are more notable in the vicinities of the end points $( \pm 1 / 3$, $1,0)$ of the zone between the QDs.

The variations of the out-of-plane component of the electric displacement field, $D_{3}$, and the in-plane and outof-plane electric field, respectively, $E_{1}$ and $E_{3}$ along the $x_{1}$-axis are shown in Figure 7. As it is seen, in the vicinities of the QD-barrier interface $( \pm 1 / 3,0,0)$, the electric field component, $E_{1}$ exhibits large variations, and more severely, the electric field component, $E_{3}$, and the electric displacement component, $D_{3}$, display a jump discontinuously at $( \pm 1 / 3,0,0)$. Although the variations of $E_{3}$ and $D_{3}$ are moderate along the $x_{1}$-axis within the base of the QD, $E_{1}$ shows a notable variation within the mentioned region.

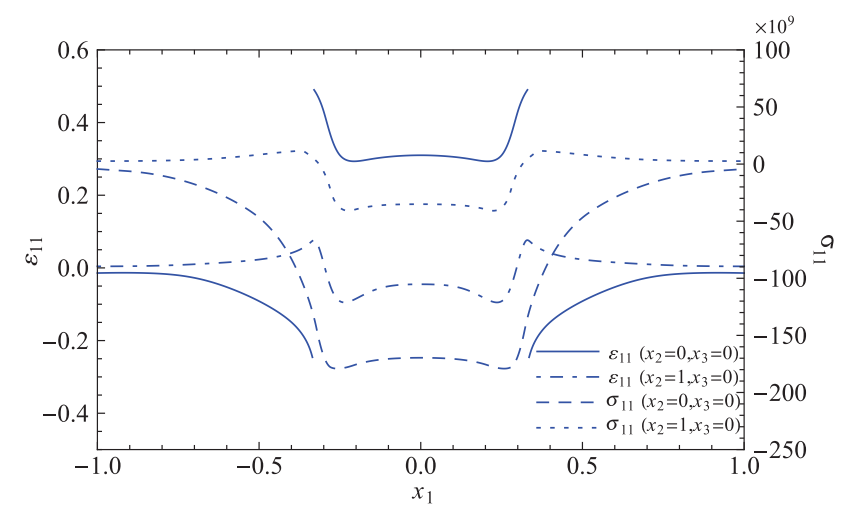

Figure 6 Variations of the total strain component, $\varepsilon_{11}$, and stress component, $\sigma_{11}$, with $x_{1}$ within the $x_{3}=0$ plane along $x_{2}=0\left(x_{1}\right.$-axis $)$ and along $x_{2}=1$. 


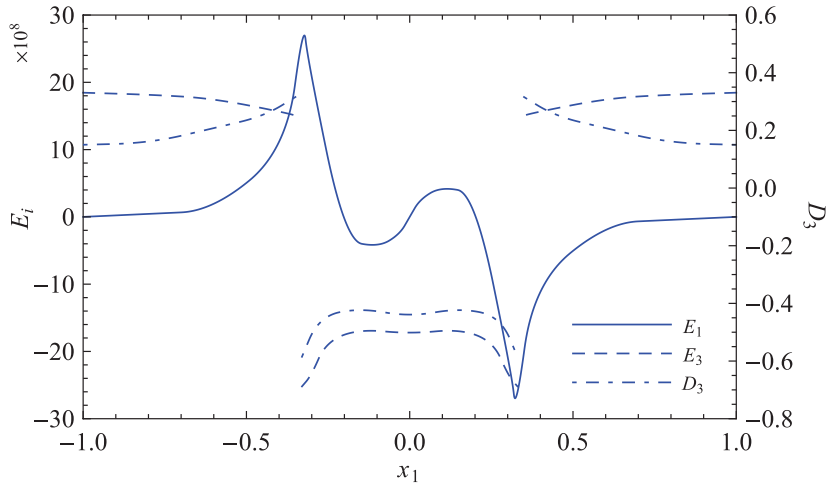

Figure 7 Variations of the total electric field, $E_{i}, i=1,3$, and electric displacement component, $D_{3}$, along the $x_{1}$-axis.

\subsection{Disturbance of the electro-mechanical fields of a single pyramidal InN QD in AIN barrier by another nearby QD}

This last example intends to address the fundamental question of the disturbance in the electro-mechanical fields of a single QD caused by another nearby QD. The geometries of the considered single pyramidal InN QD and two interacting pyramidal InN QDs within AlN barrier are depicted in Figure 8A and B, respectively. The pyramids' heights are assumed to be $1 / 3$ and the dimensions of their quadrangular bases are set equal to $2 / 3 \times 2 / 3$. The $x_{3}$-axis is taken as the vertical axis of the QDs; moreover, the direction of polarization and the axis of the rotational symmetry of the QDs and the barrier are all assumed to be parallel to the $x_{3}$-axis.

The relative difference between the lattice parameters of InN QD and AlN barrier is about 0.14 [16]. Thus, assuming the dilatational eigenstrain field

$$
\varepsilon_{i j}^{p}=0.14 \delta_{i j},
$$

within the QDs, it can be shown that the exact expression for the Fourier coefficient, $\bar{\varepsilon}_{i j}^{p}(\xi)$, is

$$
\begin{aligned}
\bar{\varepsilon}_{i j}^{p}(\xi)= & \frac{0.14}{4 \pi^{3} \xi_{1} \xi_{2}}\left[-\frac{4 i e^{\frac{i \xi_{3}}{3}} \xi_{1} \xi_{2} \xi_{3}}{\xi_{1}^{4}+\left(\xi_{2}^{2}-\xi_{3}^{2}\right)^{2}-2 \xi_{1}^{2}\left(\xi_{2}^{2}+\xi_{3}^{2}\right)}\right. \\
& +\frac{i \xi_{3} \operatorname{Cos}\left[\frac{1}{3}\left(-\xi_{1}+\xi_{2}\right)\right]+\left(\xi_{1}-\xi_{2}\right) \operatorname{Sin}\left[\frac{\xi_{1}-\xi_{2}}{3}\right]}{\left(\xi_{1}-\xi_{2}\right)^{2}-\xi_{3}^{2}} \\
& \left.-\frac{\left.i \xi_{3} \operatorname{Cos}\left[\frac{\xi_{1}+\xi_{2}}{3}\right]+\left(\xi_{1}+\xi_{2}\right) \operatorname{Sin}\left[\frac{\xi_{1}+\xi_{2}}{3}\right]\right]}{\left(\xi_{1}+\xi_{2}-\xi_{3}\right)\left(\xi_{1}+\xi_{2}+\xi_{3}\right)}\right] \delta_{i j},
\end{aligned}
$$

for the single QD and

$$
\begin{aligned}
\bar{\varepsilon}_{i j}^{p}(\xi)= & \frac{0.14}{4 \pi^{3} \xi_{1} \xi_{2}}\left[e^{\frac{i \xi_{3}}{3}}\left(\frac{i \xi_{3}}{\left(\xi_{1}+\xi_{2}-\xi_{3}\right)\left(\xi_{1}+\xi_{2}+\xi_{3}\right)}-\frac{i \xi_{3}}{\left(\xi_{1}-\xi_{2}\right)^{2}-\xi_{3}^{2}}\right)\right. \\
& -e^{\frac{2 i \xi_{3}}{3}\left(\frac{-i \xi_{3} \operatorname{Cos}\left[\frac{\xi_{2}-\xi_{1}}{3}\right]+\left(\xi_{1}-\xi_{2}\right) \operatorname{Sin}\left[\frac{\xi_{2}-\xi_{1}}{3}\right]}{\left(\xi_{1}-\xi_{2}\right)^{2}-\xi_{3}^{2}}\right)} \\
& +\frac{\left.i \xi_{3} \operatorname{Cos}\left[\frac{\xi_{1}+\xi_{2}}{3}\right]+\left(\xi_{1}+\xi_{2}\right) \operatorname{Sin}\left[\frac{\xi_{1}+\xi_{2}}{3}\right]\right)}{\left(\xi_{1}+\xi_{2}-\xi_{3}\right)\left(\xi_{1}+\xi_{2}+\xi_{3}\right)} e^{\frac{i \xi_{3}}{3}} \frac{4 i \xi_{1} \xi_{2} \xi_{3}}{\xi_{1}^{4}+\left(\xi_{2}^{2}-\xi_{3}^{2}\right)^{2}-2 \xi_{1}^{2}\left(\xi_{2}^{2}+\xi_{3}^{2}\right)} \\
& +e^{\frac{2 i \xi_{3}}{3}}\left(\frac{-i \xi_{3} \operatorname{Cos}\left[\frac{\xi_{1}-\xi_{2}}{3}\right]+\left(\xi_{1}-\xi_{2}\right) \operatorname{Sin}\left[\frac{\xi_{1}-\xi_{2}}{3}\right]}{\left(\xi_{1}-\xi_{2}\right)^{2}-\xi_{3}^{2}}\right] \\
& +\frac{\left.\left.i \xi_{3} \operatorname{Cos}\left[\frac{\xi_{1}+\xi_{2}}{3}\right]-\left(\xi_{1}+\xi_{2}\right) \operatorname{Sin}\left[\frac{\xi_{1}+\xi_{2}}{3}\right]\right)\right] \delta_{i j}}{\left(\xi_{1}+\xi_{2}-\xi_{3}\right)\left(\xi_{1}+\xi_{2}+\xi_{3}\right)}
\end{aligned}
$$
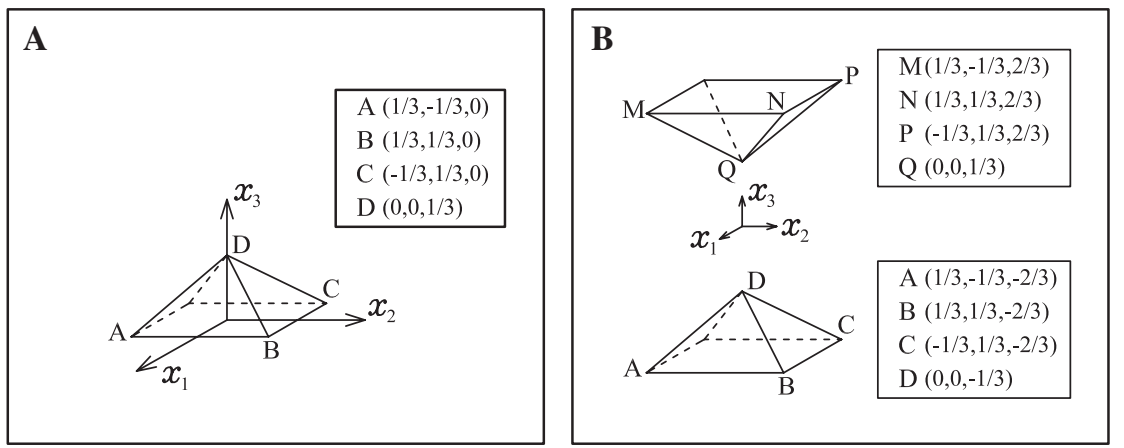

Figure 8 Geometries of (A) a single InN QD and (B) two interacting InN QDs, within AIN barrier. 
for the two interacting QDs. Figure 9 compares the variations of the total strain component, $\varepsilon_{33}$, and the stress component, $\sigma_{33}$, along the vertical axis of the QD ( $x_{3}$-axis) associated to a single QD with that of two interacting QDs. In this figure, the regions occupied by the QDs are indicated as QD-1 and QD-2, where their vertical axes are in the ranges $-2 / 3 \leq x_{3} \leq-1 / 3$ and $1 / 3 \leq x_{3} \leq 2 / 3$, respectively. In the case of a single QD, only QD-1 is considered. As it is seen, the value of $\sigma_{33}$ is always negative. The value of $\varepsilon_{33}$ is always negative in the barrier, whereas it is positive within the QDs. Inside the barrier in the vicinity of the QDs, in general, the rate of decrease in $\varepsilon_{33}$ is large, and becomes infinite at the QD-barrier interface where there is a significant jump discontinuity in $\varepsilon_{33}$. For the case of the interacting QDs for which $\varepsilon_{33}$ and $\sigma_{33}$ are symmetric with respect to $x_{3}=0$, the values of $\varepsilon_{33}$ and $\sigma_{33}$ become vanishingly small at distances larger than about four times the height of the QDs (i.e., $\left|x_{3}\right|>1.8$ ). In the case of a single QD, within the barrier, $\varepsilon_{33}$ has a larger absolute value at the QD's apex than at its quadrangular base; correspondingly, the maximum absolute value of $\sigma_{33}$ occurs at the apex of the QD. $\sigma_{33}$ is continuous everywhere, and the presence of the second QD has resulted not only in two equal local maximum absolute values of $\sigma_{33}$ at their apices but also larger maximum absolute values of $\sigma_{33}$. The absolute values of $\varepsilon_{33}$ everywhere in the region between the two apices are larger than those of a single QD, whereas the variation of $\varepsilon_{33}$ pertinent to a single QD for $-1 / 3 \leq x_{3} \leq 0$ is much larger than that of the interacting QDs.

\section{Conclusion}

The current study provides an analytical treatment for the calculation of the electro-elastic fields of a periodic

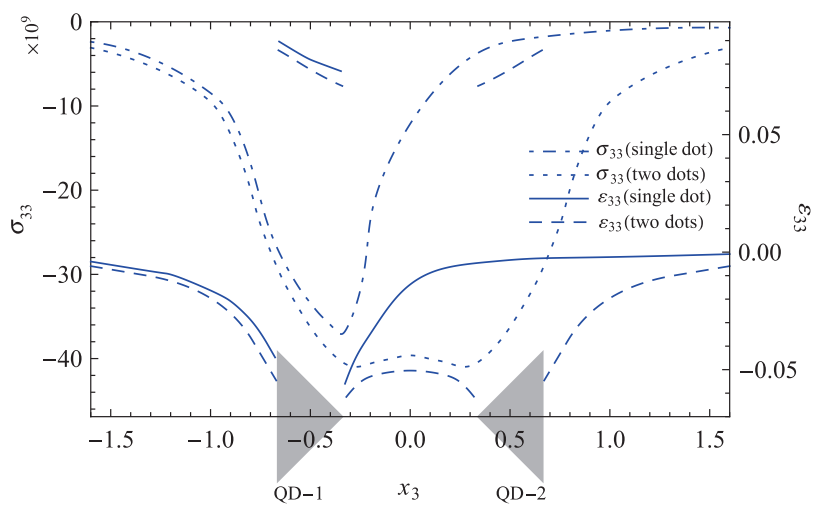

Figure 9 Variations of the total strain component $\varepsilon_{33}$ and stress component $\sigma_{33}$ along the $x_{3}$-axis corresponding to a single QD and two interacting QDs. or an arbitrary distribution of a finite number of interacting piezoelectric QWRs/QDs with arbitrary geometries within a piezoelectric barrier of yet different electromechanical properties. The QWRs/QDs may contain any arbitrary misfit strain field, and all piezoelectric media can have general anisotropy. Furthermore, this work treats the case where an FG zone is inserted between the QWRs/QDs and the barrier as well. Interestingly, under certain conditions, the initial eigenstrain and eigenelectric fields are electrically and elastically impotent, respectively (Theorems 1 and 2 and Remark 1). Proper account of the difference between the electro-mechanical properties of the QWRs/QDs and those of the barrier can lead to the improvement of the results by as much as about 8\%. Section 5.1 reconsidered a single spherical QD, which was previously examined in the literature, merely for the sake of comparison. Sections 5.2 and 5.3 demonstrated that the theory is conveniently applicable to 2D/3D periodic distributions of interacting QWRs/QDs, respectively; the high accuracy and the relative ease in handling such periodic distributions, which are far more complicated cases than the case of just a single QWR/QD, is mainly because of the formulation of the consistency equations in the Fourier space. Finally, the example in Section 5.4 reveals that the electro-mechanical fields of a single pyramidal InN QD in AlN barrier are significantly disturbed by a nearby QD.

\section{Appendix A: Components of the cofactor, $N(\xi)$, for 3D transversely isotropic piezoelectric media}

The components of the cofactor, $\mathbf{N}(\boldsymbol{\xi})$, in 3D space corresponding to transversely isotropic piezoelectric media are

$$
\begin{aligned}
& N_{11}\left(\xi_{1}, \xi_{2}, \xi_{3}\right) \\
& =\left(e_{15}+e_{31}\right) \xi_{2}^{2} \xi_{3}^{2}\left(\left(C_{13} e_{15}-C_{44} e_{31}\right)\left(\xi_{1}^{2}+\xi_{2}^{2}\right)+\left(-C_{33}\left(e_{15}+e_{31}\right)\right.\right. \\
& \left.\left.+\left(C_{13}+C_{44}\right) e_{33}\right) \xi_{3}^{2}\right)+\left(-\kappa_{11}\left(\xi_{1}^{2}+\xi_{2}^{2}\right)-\kappa_{33} \xi_{3}^{2}\right)\left(-\left(C_{13}+C_{44}\right)^{2} \xi_{2}^{2} \xi_{3}^{2}\right. \\
& \left.+\left(C_{44}\left(\xi_{1}^{2}+\xi_{2}^{2}\right)+C_{33} \xi_{3}^{2}\right)\left(\frac{1}{2}\left(C_{11}-C_{12}\right) \xi_{1}^{2}+C_{11} \xi_{2}^{2}+C_{44} \xi_{3}^{2}\right)\right) \\
& -\left(e_{15}\left(\xi_{1}^{2}+\xi_{2}^{2}\right)+e_{33} \xi_{3}^{2}\right)\left(-\left(C_{13}+C_{44}\right)\left(e_{15}+e_{31}\right) \xi_{2}^{2} \xi_{3}^{2}\right. \\
& \left.+\left(\frac{1}{2}\left(C_{11}-C_{12}\right) \xi_{1}^{2}+C_{11} \xi_{2}^{2}+C_{44} \xi_{3}^{2}\right)\left(e_{15}\left(\xi_{1}^{2}+\xi_{2}^{2}\right)+e_{33} \xi_{3}^{2}\right)\right)
\end{aligned}
$$




$$
\begin{aligned}
& N_{12}\left(\xi_{1}, \xi_{2}, \xi_{3}\right) \\
& \quad=\frac{1}{2} \xi_{1} \xi_{2}\left(\left(C_{11}+C_{12}\right)\left(e_{15}^{2}+C_{44} \kappa_{11}\right)\left(\xi_{1}^{2}+\xi_{2}^{2}\right)^{2}\right. \\
& -\left(2 C_{44}\left(e_{15}-e_{31}\right)\left(e_{15}+e_{31}\right)+2 C_{13}^{2} \kappa_{11}+2 C_{44}^{2} \kappa_{11}\right. \\
& -\left(C_{11}+C_{12}\right)\left(2 e_{15} e_{33}+C_{33} \kappa_{11}\right)+4 C_{13}\left(e_{15}\left(e_{15}+e_{31}\right)+C_{44} \kappa_{11}\right) \\
& \left.\quad-\left(C_{11}+C_{12}\right) C_{44} \kappa_{33}\right)\left(\xi_{1}^{2}+\xi_{2}^{2}\right) \xi_{3}^{2}+\left(2 C_{33}\left(e_{15}+e_{31}\right)^{2}\right. \\
& +e_{33}\left(-4\left(C_{13}+C_{44}\right)\left(e_{15}+e_{31}\right)+\left(C_{11}+C_{12}\right) e_{33}\right) \\
& \left.\left.+\left(C_{11}+C_{12}\right) C_{33} \kappa_{33}-2\left(C_{13}+C_{44}\right)^{2} \kappa_{33}\right) \xi_{3}^{4}\right), t
\end{aligned}
$$

$$
\begin{aligned}
& N_{13}\left(\xi_{1}, \xi_{2}, \xi_{3}\right) \\
& \quad=\frac{1}{2} \xi_{1} \xi_{3}\left(\left(C_{11}-C_{12}\right)\left(\xi_{1}^{2}+\xi_{2}^{2}\right)+2 C_{44} \xi_{3}^{2}\right)\left(\left(e_{15}\left(e_{15}+e_{31}\right)\right.\right. \\
& \left.\quad+\left(C_{13}+C_{44}\right) \kappa_{11}\right)\left(\xi_{1}^{2}+\xi_{2}^{2}\right)+\left(\left(e_{15}+e_{31}\right) e_{33}\right. \\
& \left.\left.\quad+\left(C_{13}+C_{44}\right) \kappa_{33}\right) \xi_{3}^{2}\right),
\end{aligned}
$$

$$
\begin{aligned}
& N_{14}\left(\xi_{1}, \xi_{2}, \xi_{3}\right) \\
& =\frac{1}{2} \xi_{1} \xi_{3}\left(\left(C_{11}-C_{12}\right)\left(\xi_{1}^{2}+\xi_{2}^{2}\right)+2 C_{44} \xi_{3}^{2}\right)\left(\left(C_{13} e_{15}-C_{44} e_{31}\right)\left(\xi_{1}^{2}+\xi_{2}^{2}\right)\right. \\
& \left.+\left(-C_{33}\left(e_{15}+e_{31}\right)+\left(C_{13}+C_{44}\right) e_{33}\right) \xi_{3}^{2}\right),
\end{aligned}
$$

$$
\begin{aligned}
& N_{22}\left(\xi_{1}, \xi_{2}, \xi_{3}\right) \\
& \quad=\left(e_{15}+e_{31}\right) \xi_{1}^{2} \xi_{3}^{2}\left(\left(C_{13} e_{15}-C_{44} e_{31}\right)\left(\xi_{1}^{2}+\xi_{2}^{2}\right)+\left(-C_{33}\left(e_{15}+e_{31}\right)\right.\right. \\
& \left.\left.+\left(C_{13}+C_{44}\right) e_{33}\right) \xi_{3}^{2}\right)+\left(-\kappa_{11}\left(\xi_{1}^{2}+\xi_{2}^{2}\right)-\kappa_{33} \xi_{3}^{2}\right)\left(-\left(C_{13}+C_{44}\right)^{2} \xi_{1}^{2} \xi_{3}^{2}\right. \\
& \left.+\left(C_{44}\left(\xi_{1}^{2}+\xi_{2}^{2}\right)+C_{33} \xi_{3}^{2}\right)\left(C_{11} \xi_{1}^{2}+\frac{1}{2}\left(C_{11}-C_{12}\right) \xi_{2}^{2}+C_{44} \xi_{3}^{2}\right)\right) \\
& -\left(e_{15}\left(\xi_{1}^{2}+\xi_{2}^{2}\right)+e_{33} \xi_{3}^{2}\right)\left(-\left(C_{13}+C_{44}\right)\left(e_{15}+e_{31}\right) \xi_{1}^{2} \xi_{3}^{2}\right. \\
& \left.+\left(C_{11} \xi_{1}^{2}+\frac{1}{2}\left(C_{11}-C_{12}\right) \xi_{2}^{2}+C_{44} \xi_{3}^{2}\right)\left(e_{15}\left(\xi_{1}^{2}+\xi_{2}^{2}\right)+e_{33} \xi_{3}^{2}\right)\right),
\end{aligned}
$$

$$
\begin{aligned}
& N_{23}\left(\xi_{1}, \xi_{2}, \xi_{3}\right) \\
& \quad=\frac{1}{2} \xi_{2} \xi_{3}\left(\left(C_{11}-C_{12}\right)\left(\xi_{1}^{2}+\xi_{2}^{2}\right)+2 C_{44} \xi_{3}^{2}\right)\left(\left(e_{15}\left(e_{15}+e_{31}\right)\right.\right. \\
& \left.\left.+\left(C_{13}+C_{44}\right) \kappa_{11}\right)\left(\xi_{1}^{2}+\xi_{2}^{2}\right)+\left(\left(e_{15}+e_{31}\right) e_{33}+\left(C_{13}+C_{44}\right) \kappa_{33}\right) \xi_{3}^{2}\right),
\end{aligned}
$$

$$
\begin{aligned}
& N_{24}\left(\xi_{1}, \xi_{2}, \xi_{3}\right) \\
& =\frac{1}{2} \xi_{2} \xi_{3}\left(\left(C_{11}-C_{12}\right)\left(\xi_{1}^{2}+\xi_{2}^{2}\right)+2 C_{44} \xi_{3}^{2}\right)\left(\left(C_{13} e_{15}-C_{44} e_{31}\right)\left(\xi_{1}^{2}+\xi_{2}^{2}\right)\right. \\
& \left.+\left(-C_{33}\left(e_{15}+e_{31}\right)+\left(C_{13}+C_{44}\right) e_{33}\right) \xi_{3}^{2}\right),
\end{aligned}
$$

$$
\begin{aligned}
& N_{33}\left(\xi_{1}, \xi_{2}, \xi_{3}\right) \\
& =-\frac{1}{2}\left(\left(C_{11}-C_{12}\right)\left(\xi_{1}^{2}+\xi_{2}^{2}\right)+2 C_{44} \xi_{3}^{2}\right)\left(C_{11} \kappa_{11}\left(\xi_{1}^{2}+\xi_{2}^{2}\right)^{2}\right. \\
& \left.+\left(\left(e_{15}+e_{31}\right)^{2}+C_{44} \kappa_{11}+C_{11} \kappa_{33}\right)\left(\xi_{1}^{2}+\xi_{2}^{2}\right) \xi_{3}^{2}+C_{44} \kappa_{33} \xi_{3}^{4}\right), \\
& N_{34}\left(\xi_{1}, \xi_{2}, \xi_{3}\right) \\
& \quad=-\frac{1}{2}\left(\left(C_{11}-C_{12}\right)\left(\xi_{1}^{2}+\xi_{2}^{2}\right)+2 C_{44} \xi_{3}^{2}\right)\left(C_{11} e_{15}\left(\xi_{1}^{2}+\xi_{2}^{2}\right)^{2}\right. \\
& \left.\quad-\left(C_{44} e_{31}+C_{13}\left(e_{15}+e_{31}\right)-C_{11} e_{33}\right)\left(\xi_{1}^{2}+\xi_{2}^{2}\right) \xi_{3}^{2}+C_{44} e_{33} \xi_{3}^{4}\right), \\
& N_{44}\left(\xi_{1}, \xi_{2}, \xi_{3}\right) \\
& =\frac{1}{2}\left(\left(C_{11}-C_{12}\right)\left(\xi_{1}^{2}+\xi_{2}^{2}\right)+2 C_{44} \xi_{3}^{2}\right)\left(C_{11} C_{44}\left(\xi_{1}^{2}+\xi_{2}^{2}\right)^{2}\right. \\
& \left.\quad-\left(C_{13}^{2}-C_{11} C_{33}+2 C_{13} C_{44}\right)\left(\xi_{1}^{2}+\xi_{2}^{2}\right) \xi_{3}^{2}+C_{33} C_{44} \xi_{3}^{4}\right) .
\end{aligned}
$$

\section{References}

[1] Mo YW, Savage DE, Swartzentruber BS, Lagally MG. Phys. Rev. Lett. 1990, 65, 1020-1023.

[2] Grundmann M, Christen J, Ledentsov NN, Bohrer J, Bimberg D, Ruvimov SS, Werner P, Richter U, Gosele U, Heydenreich J, Ustinov VM, Egorov AY, Zhukov AE, Kop'ev PS, Alferov ZI. Phys. Rev. Lett. 1995, 74, 4043-4046.

[3] Jin G, Tang YS, Liu JL, Wang KL. Appl. Phys. Lett. 1999, 74, 2471-2473.

[4] Jacak L. Eur. J. Phys. 2000, 21, 487-497.

[5] Grundmann M, Stier O, Bimberg D. Phys. Rev. B 1995, 52, 11969-11981.

[6] Faux DA, Downes JR, O’Reilly EP. J. Appl. Phys. 1997, 82, 3754-3762.

[7] Davies JH. J. Appl. Phys. 1998, 84, 1358-1365.

[8] Faux DA, Pearson GS. Phys. Rev. B 2000, 62, R4798-R4801.

[9] Pan E. J. Appl. Phys. 2002, 91, 3785-3796.

[10] Eshelby JD. Prog. Solid Mech. 1961, 2, 89-140.

[11] Eshelby JD. Proc. R. Soc. A 1957, 241, 376-396.

[12] Shokrolahi-Zadeh B, Shodja HM. J. Mech. Phys. Solids 2008, 56, 3565-3575.

[13] Shodja HM, Khorshidi A. J. Mech. Phys. Solids 2013, 61, 1124-1143.

[14] Markenscoff X, Dundurs J. J. Mech. Phys. Solids 2014, 64, 468-482.

[15] Singh J. Physics of Semiconductors and Their Heterostructures. McGraw-Hill: New York, 1993.

[16] Jogai B. J. Appl. Phys. 2001, 90, 699-704. 